\title{
Realization of Broadband Matched Filter Structures Based on Dual Networks
}

\author{
M. Gerding and B. Schiek
}

RF and Microwave Institute, Ruhr-University Bochum, Universitätsstrasse 150, 44780 Bochum, Germany

\begin{abstract}
This paper deals with the basic electrical properties of dual networks and with their application in broadband matched filter structures.

Starting with the main characteristics and different realization methods of dual networks, a filter structure is presented, which is based on a combination of dual networks and which provides a broadband matched input and two decoupled output ports. This filter synthesis focuses on the design of high pass filters, which are suitable to be used as differentiating stages in electrical pulse generators as a part of the so-called pulse shaping network. In order to achieve a proper pulse shape and for the prevention of multiple reflections between the switching circuit and the differentiating network, a broadband matched filter is a basic requirement.
\end{abstract}

\section{Introduction}

The transmission and reflection characteristics of dual networks offer the possibility to build filter structures, which are matched frequency-independently or at least over a broad band with respect to the input. These filters structures contain a parallel connection of two dual networks, using their frequency-independent reciprocal input impedances to achieve the broadband input matching of the resulting filter circuit. The decoupling of both output ports of the resulting filter structure is an additional feature of these structures. All this basic features are independent of the chosen filter characteristics of the dual networks and of their realization method. They can be realized by using concentrated elements, as well as planar transmission line elements. Even a mixture of both techniques is possible, without influencing their duality. Especially for the use as a differentiating stage in pulse shaping networks, a high-pass filter structure is presented, which is based on coupled line structures. Beside the basic properties of a broadband matched input and the two decoupled outputs, this structure additionally provides two identical pulse signals with opposite polarities at their outputs.

Correspondence to: M. Gerding

(michael.gerding@ruhr-uni-bochum.de)

\section{Basics of dual networks}

Dual networks are the basis for the implementation of broadband matched filters, which are presented in the following. The special features of dual networks are their identical transfer characteristics concerning the magnitude and the phase and their reciprocal input impedances. Expressed by the scattering parameters of two dual networks $N$ and $\tilde{N}$ this leads to the following relations (Matsumoto, 1970; Zverev, 1967):

$S_{21}(s)=\tilde{S}_{21}(s) \quad$ and $\quad S_{11}(s)=-\tilde{S}_{11}(s)$,

where $S_{21}, \tilde{S}_{21}$ are the forward transmission coefficients of the networks $N, \tilde{N}$, respectively and $S_{11}, \tilde{S}_{11}$ are the reflection coefficients. The relations in equation 1 lead to the reciprocal input impedances $W_{i n}(s)$ and $\tilde{W}_{i n}(s)$ :

$$
W_{i n}(s)=\frac{1+S_{11}(s)}{1-S_{11}(s)} \quad \text { and } \quad \tilde{W}_{i n}(s)=W_{i n}(s)^{-1}=\frac{1-\tilde{S}_{11}(s)}{1+\tilde{S}_{11}(s)} .
$$

The Eqs. (1) and (2) are valid for all complex frequencies $s=j \omega$.

Further properties of dual networks can be evaluated by regarding the two basic dual filter structures, which consist of LC-cascade circuits as it is illustrated in Fig. 1.

Comparing the dual structures a) and b) it is obvious, that a series element and a parallel element in one structure result in a parallel element and a series element, respectively, with the reciprocal impedance in the dual structure. This results in the following reciprocal element-pairs according to the example in Fig. 1:

$Z_{0}^{2} C_{1}=L_{1} \quad, \quad Z_{0}^{2} C_{2}=L_{2} \quad, \quad Z_{0}^{2} C_{3}=L_{3}$.

The reactances or the so called two-terminal-elements (TTE) can be built with concentrated elements as well as with planar transmission line elements. In correspondence to the two-wire equivalent circuits in Fig. 2 an inductance can be realized as a shorted stub line with the electrical length of $\beta l$ and the wave impedance $Z_{L}$ (Fig. 2a) and a capacitance can be realized as an open ended stub line with the electrical length of $\beta l$ and the wave impedance $Z_{C}$ (Fig. 2b), respectively. This effects a change of the dependency of the complex frequency to $s=j \tan \beta l$. Even a mixture 

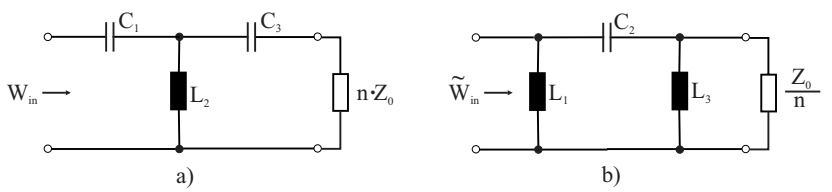

Fig. 1. Dual high-pass filters realized as LC-cascade circuits ( $\mathrm{n}$ is a positive integer, including $n=0$ ).

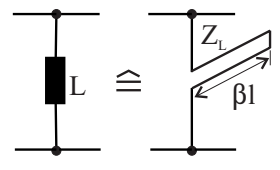

a)

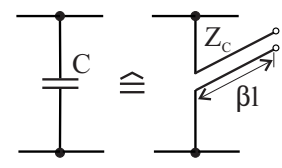

b)
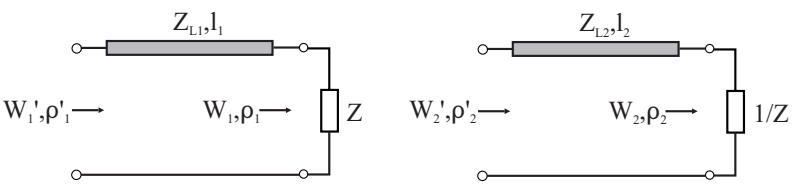

Fig. 4. Additionally inserted transmission-line-elements.
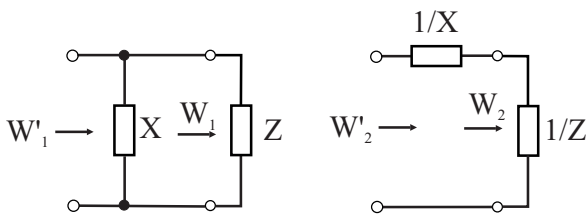

a)
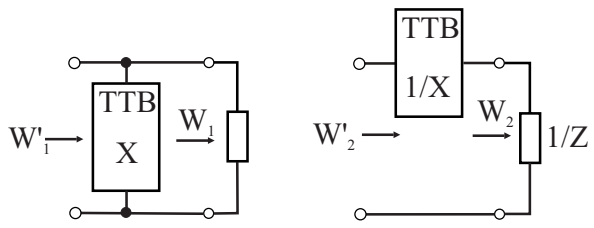

b)

Fig. 5. Additionally inserted reactances (a) and two-terminalblocks (b).

filter-circuits is not affected. This behavior concerning the duality of the filter networks can be explained as illustrated in Fig. 4. From now on, for convenience, the impedances $Z, W$, and $X$ are normalized to the reference impedance $Z_{0}$. Starting with the load impedances $W_{1}(s)$ and $W_{2}(s)$ of two dual network structures and their reciprocal relationship $W_{1}(s)=W_{2}(s)^{-1}$ and their reflection coefficients $\rho_{1}=-\rho_{2}$, respectively, the resulting reflection coefficients $\rho_{1}^{\prime}$ and $\rho_{2}^{\prime}$ of the expanded circuits can be calculated as follows. By use of the conditions above, the resulting reflection coefficients are:

$\rho_{1}^{\prime}=e^{j 2 \beta l_{1}} \rho_{1} \quad$ and $\quad \rho_{2}^{\prime}=e^{j 2 \beta l_{2}} \rho_{2}=e^{j 2 \beta l_{2}}\left(-\rho_{1}\right)$.

Because of the identical electrical length of both inserted transmission-line-elements $\beta l_{1}=\beta l_{2}=\beta l$, the exponential terms in equation 4 become equal which leads to the expression $\rho_{1}^{\prime}=-\rho_{2}^{\prime}$. This again complies with the conditions of dual circuits and with $W_{1}^{\prime}=1 / W_{2}^{\prime}$.

When realizing the two-terminal-elements in Fig. 3 as stub lines in combination with additionally inserted transmissionline-elements of the same electrical length, the use of Kuroda identities becomes possible (Malherbe, 1979), which may lead to a variety of different circuit structures.

According to the example of the dual filter structures in Fig. 1, the statement in Eq. 3 can be generalized.

Starting with any dual network, represented by its reciprocal input impedances $W_{1}(s)$ and $W_{2}(s)$ in Fig. 5 a), the insertion of any reciprocal two-terminal-element, e.g. $X$ or $1 / X$ 


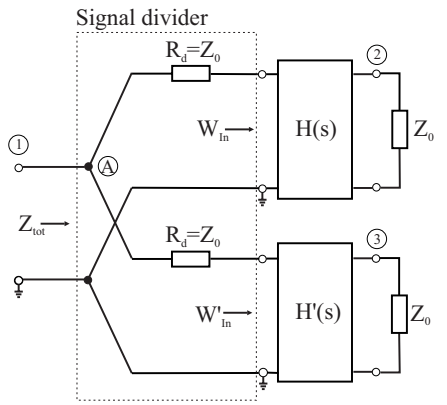

a)

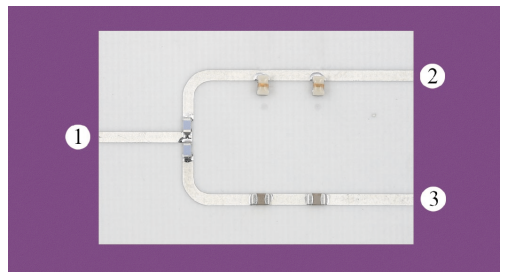

b)

Fig. 6. Broadband matched filter structure: (a) in principle, (b) realization with concentrated elements.
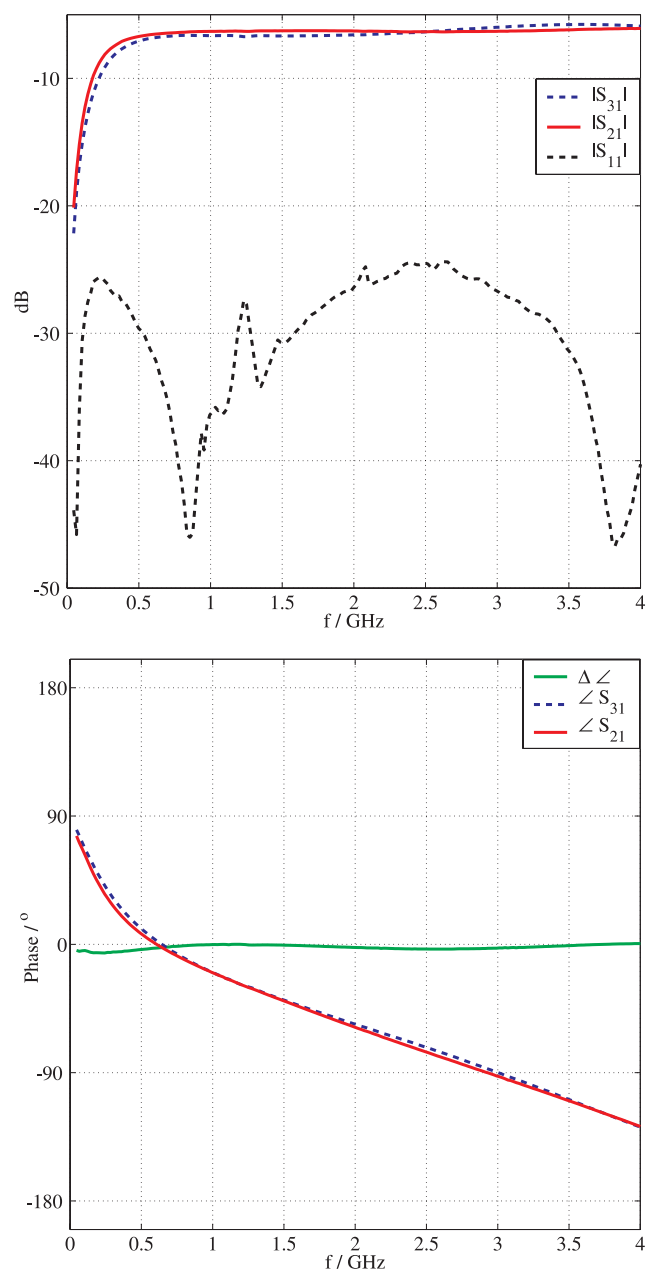

Fig. 7. Measurement results of the filter structure in Fig. 6 b.
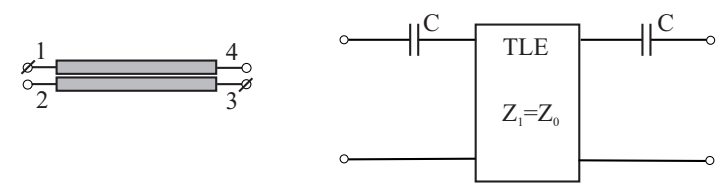

a)
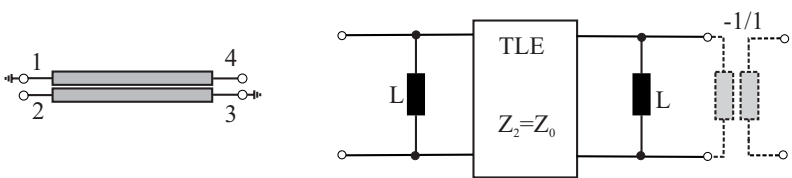

b)

Fig. 8. Coupled line structures with their corresponding equivalent circuits.

in parallel or in series, respectively, will again lead to dual networks, which can be evaluated by calculating the input impedances $W_{1}^{\prime}$ and $W_{2}^{\prime}$, which are reciprocal, as is expected.

$W_{1}^{\prime}(s)=\frac{1}{\frac{1}{Z}+\frac{1}{X}} \quad$ und $\quad W_{2}^{\prime}(s)=\frac{1}{Z}+\frac{1}{X}=\left(W_{1}^{\prime}(s)\right)^{-1}$

In a further generalization the two-terminal-elements $X$ and $1 / X$ can be represented by the input impedances of complex dual networks, the so called two-terminal-blocks (TTB) in Fig. 5b, as well.

\section{The realization of broadband matched filter struc- tures}

In some applications an input mismatch in the stop band of a filter network is not tolerable. Therefore frequencyindependent or at least broadband matched filters are necessary. The following description is focused on a broadband matched filter structure, especially for the use as a differentiating stage in electrical pulse generators. 


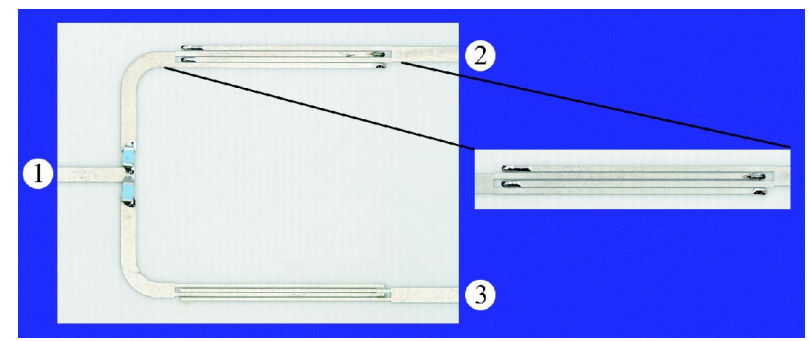

Fig. 9. Realization of a broadband matching filter structure, based on dual coupled line pairs.
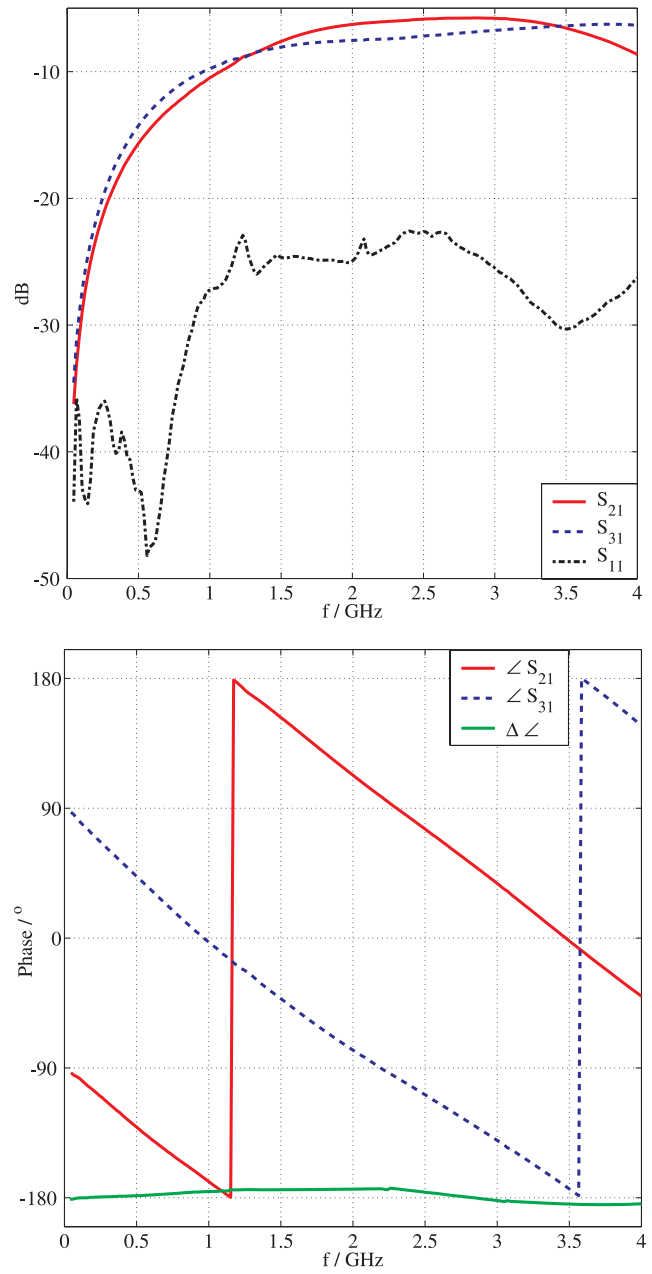

Fig. 10. Measurement results of the filter structure in Fig. 9.

\subsection{Broadband matched filters based on dual networks}

Dual structures and especially their reciprocal input impedances are the basis for the broadband matched filters, which are presented in the following. An exemplary broadband matched filter structure is shown in Fig. 6a in principle, which basically consists of two dual networks $H(s)$ and $H^{\prime}(s)$ in parallel combined by a signal divider circuit. By evaluating the input impedance $Z_{t o t}(s)$ it can be shown that

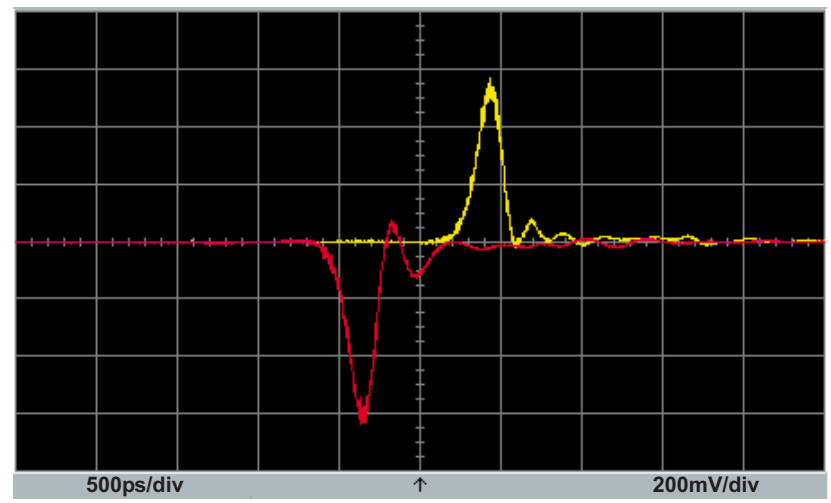

Fig. 11. Measurement of the output signals of the dual coupled line filter structure in Fig. 9 (The signal delay is caused by different line length in the measurement setup.).

the structure is frequency-independently matched at its input. $Z_{t o t}(s)$ can be calculated as:

$$
\begin{aligned}
Z_{\text {tot }}(s) & =\left(1+W_{i n}(s)\right) \|\left(1+W_{i n}^{\prime}(s)\right) \\
& =\left(\frac{1}{1+W_{i n}(s)}+\frac{1}{1+W_{i n}^{\prime}(s)}\right)^{-1}=1 \\
& \text { with } \quad W_{i n}(s)=1 / W_{i n}^{\prime}(s) .
\end{aligned}
$$

Beside the broadband input matching, the decoupling of the two filters is a second main characteristic of this structure. Because of the reciprocal input impedances $W_{i n}(s)$ and $W_{i n}^{\prime}(s)$ and the resulting reflection coefficients with opposite signs of the dual networks $H$ and $H^{\prime}$, point A in Fig. 6 can be interpreted as a virtual ground. The circuit shows an additional signal attenuation of $6 \mathrm{~dB}$.

Figure $6 \mathrm{~b}$ shows an implementation of a broadband matched high-pass filter network, including the dual networks, which are realized with concentrated reactances and transmissionline-elements for expanding the structure. The corresponding measurement results are illustrated in Fig. 7. The magnitude and the phase of the scattering parameters $S_{21}$ and $S_{31}$ are nearly equal, in correspondence to Eq. 1, which leads to nearly identical output signals at the ports 2 and 3 and furthermore the input matching versus frequency at port 1 is better than $-25 \mathrm{~dB}$, as expected.

\subsection{Signal inverting broadband matched filters}

Based on the network principle of Fig. 6a the filter network can also be implemented by using proper coupled line structures instead of the conventional dual networks $H$ and $H^{\prime}$. With the use of the corresponding equivalent circuits and the Kuroda identities (Malherbe, 1979) in Fig. 8 it can be shown that an open ended coupled line structure a) and a shorted ended coupled line structure b) can behave like dual networks, considering the properties of dual circuits mentioned above. According to this the inductances and capacitances of the equivalent circuits must be reciprocal to each other, which can be achieved by choosing the width of the coupled 
lines and their distances accordingly (Kirschning, 1984). Furthermore the wave impedances of the transmission-lineelements have to be equal to the reference impedance $Z_{0}$. The transformer in the equivalent circuit in Fig. $8 \mathrm{~b}$ is due to the change of the reference potential, which is caused by the different line terminations and which leads to a frequencyindependent phase shift of $180^{\circ}$ between the two output signals of the resulting filter structure. The corresponding measurements of the scattering parameters $S_{21}$ and $S_{31}$ are shown in Fig. 10. The rather constant and frequency-independent phase shift of about $180^{\circ}$ between the two output signals at the ports 2 and 3 is of special interest, when using this filter structure as a differentiating network in electrical pulse generators.

It offers the possibility of creating two identical pulse signals with different polarities with only one active pulse generator network (Gerding, 2003). This reduces the circuitry, e.g. in diode sampling mixers, which need two pulse signals with different polarities for driving the sampling mixer structure. Figure 11 shows an exemplary measurement in the time domain of the two output signals of the high-pass filter structure, working as a differentiating stage in a pulse generator. To achieve a still better equality of both transfer functions and of the pulse shapes, respectively, a fine tuning with an additional numerical circuit optimization is necessary, in order to optimize the behavior of the coupled line circuits in Fig. 9.

\section{Conclusion}

Starting with the basic principles of dual circuits the synthesis of broadband matched filters based on dual networks is presented in this paper. The resulting 3-port filter structures are a kind of parallel connection of two dual networks, using their frequency-independent reciprocal input impedances as a key component for achieving a broadband input matching and providing two decoupled output ports, which prevents multiple reflections and crosstalk between the two filter paths. Beside an identical transfer characteristic of each filter path, resulting in two identical output signals, it is even possible to design dual networks which cause a phase shift of $180^{\circ}$ between the two output signals. Especially for the use as a differentiating stage in electrical pulse generators, this solution gives the opportunity of generating two identical electrical pulses with opposite polarities with passive circuits and only one active pulse generator network.

\section{References}

Coekin, J.A.: High-Speed Pulse Techniques, Pergamon Press, New York 1975

Gerding, M., Musch, T., and Schiek, B.: Generation of short electrical pulses based on bipolar transistors, Kleinheubacher Berichte 2003, Ad. Radio Sci., 7-12 2004.

Kirschning, M., and Jansen, R. H.: Accurate Wide-Range Design Equations for the Frequency-Dependent Characteristic of Parallel Coupled Microstrip Lines, IEEE Transactions on Microwave Theory and Techniques, Vol. MTT-32, 83, 1984.

Malherbe, J. A. G.: Microwave Transmission Line Filters Artech House, 1979.

Malherbe, J.A.G.: Microwave Transmission Line Couplers Artech House, 1988

Matsumoto, A.: Microwave Filters and Circuits Academic Press, 1970.

Pfeiffer, W.: Impulstechnik, Carl Hander Verlag München, 1976.

Schiek, B.: Grundlagen der Hochfrequenz-Messtechnik, Springer Verlag, 1999.

Zverev, A. I.: Handbook of Filter Synthesis, John Wiley and Sons, New York, 1967. 